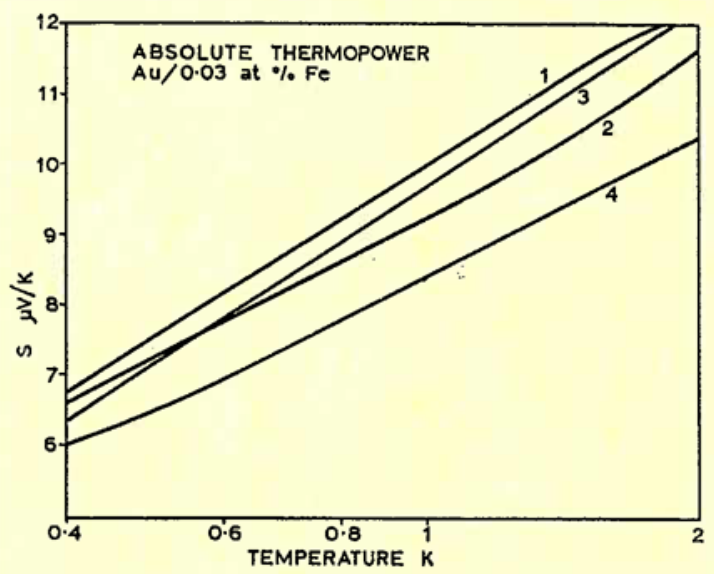

Fig. 3 Calibration of three $\mathrm{Au}+0.03$ at. $\%$ Fe vs. superconductor thermocouples below 2K. Approximate years of manufacture:

1-1966, 2-1967, 3-1969, 4-1970

From all these experiments we may conclude that the calibration of a gold-iron wire used in a thermocouple is exceedingly stable during repeated temperature cycling and is little affected by normal mechanical handling. As the sensitivity down to $\sim 1 \mathrm{~K}$ is comparable with that of thermocouples in common use at normal temperatures, there are many low temperature applications where they can be conveniently used, employing standard electrical measurement techniques. The differences between different batches of wire are sufficient to require checking of the calibration at a few conveniently realised fixed points if a temperature difference is required to be known to better than about 1 per cent. However, all the calibrations seem to differ from one another in a smooth manner so that adjustments are not difficult to make.

\section{References}

1 See, for example, J. K. Hulm, Cryogenics, 1972, 12, 472

2 G. Borelius, W. H. Keesom, C. H. Johansson and J. O. Linde, Proc. Kon. Akad. Amsterdam, 1932, 35, 10

3 W. B. Pearson and I. M. Templeton, Canad. F. Phys, 1961, 39, 1084; D. K. C. MacDonald, W. B. Pearson and I. M. Templeton, Proc. Roy. Soc., 1962, A266, 161

4 R. Berman and D. J. Huntley, Cryogenics, 1963, 3, 70 R. Berman, J. C. F. Brock and D. J. Huntley, Cryogenics, $1964,4,233$

5 R. Berman and J. Kopp, F. Phys. F: Metal Phys., 1971, 1, 457

6 L. L. Sparks and R. L. Powell, Measurements and Data 1967, 1, 82; F. Res. Nat. Bur. Standards, 1972, 76A, 263

7 R. L. Rosenbaum, Rev. Sci. Instrum., 1968, 39, 890; ibid., 1969, 40, 577

8 B. W. A. Ricketson, Private communication

9 D. P. Goulder, Private communication

\title{
A Low Temperature Resistance Thermometer
}

The well-known platinum resistance thermometer - widely used in industry and accepted for part of the International Temperatures Scale over the range from $13.8 \mathrm{~K}$ to the ice point-has disadvantages at very low temperatures in that the resistance to be measured is then small, while it is inconvenient to measure resistances with values spread over three decades.

The addition of small amounts of transition metals to gold has an anomalous effect on its resistivity at low temperatures, and on this basis a new type of cryogenic resistance thermometer has been developed by Degussa of Hanau, West Germany. This employs a 0.4 per cent manganese-gold alloy which has proved to be entirely stable and can be drawn into fine wire.

The addition of this small amount of manganese to gold is dramatic in that the resistance of a sensor that is $50 \mathrm{ohms}$ at the ice point falls to $25 \mathrm{ohms}$ at helium temperatures rather than to $0.01 \mathrm{ohm}$ with pure gold. Most of this additional resistance is temperature independent, but the alloy still has a positive slope of resistance against temperature from a value that is probably as low as $0.1 \mathrm{~K}$.

The detection voltage for any given accuracy of measurement is dependent on the maximum current that may be passed through the sensor without causing the wire to overheat appreciably and on the value of the fractional sensitivity $\mathrm{dR} / \mathrm{RdT}$. The maximum current for this new thermometer in any particular thermal environment is likely to be similar to that of platinum thermometers of the same resistance and construction. The fractional sensitivity is better than that of the nickel-manganin strain gauge type of sensor and appreciably the same as that of the gallium-arsenide diode. It is at its highest around $2 \mathrm{~K}$, but falls rapidly by nearly a magnitude between 10 and $15 \mathrm{~K}$.

Linearisation, an important electronic process that allows a display of temperature from sensor measurement, should be comparatively easy between $40 \mathrm{~K}$ and the ice point as the sensitivity alters by only about 10 per cent.

If the reproducibility of this sensor-marketed under the name Keltip-proves to be high with time and thermal cycling, a very useful new instrument will have become available for both industrial and laboratory use, particularly where millikelvin accuracy is not essential but where simple and multiple presentation of temperature is required.

B. W. A. R. 